\title{
An Efficient Scheme for Proving a Shuffle
}

\author{
Jun Furukawa and Kazue Sako \\ NEC Corporation, 4-1-1 Miyazaki, Miyamae, Kawasaki 216-8555, Japan \\ j-furukawa@ay.jp.nec.com, k-sako@ab.jp.nec.com
}

\begin{abstract}
In this paper, we propose a novel and efficient protocol for proving the correctness of a shuffle, without leaking how the shuffle was performed. Using this protocol, we can prove the correctness of a shuffle of $n$ data with roughly $18 n$ exponentiations, where as the protocol of Sako-Kilian[SK95] required $642 n$ and that of Abe Ab99 required $22 n \log n$. The length of proof will be only $2^{11} n$ bits in our protocol, opposed to $2^{18} n$ bits and $2^{14} n \log n$ bits required by Sako-Kilian and Abe, respectively. The proposed protocol will be a building block of an efficient, universally verifiable mix-net, whose application to voting system is prominent.
\end{abstract}

Keywords: Mix-net, Permutation, Electronic Voting, Universal Verifiability

\section{Introduction}

A mix-net Ch81] scheme is useful for applications which require anonymity, such as voting. The core technique in a mix-net scheme is to execute multiple rounds of shuffling and decryption by multiple, independent mixers, so that the output decryption can not be linked to any of the input encryptions.

To ensure the correctness of the output, it is desirable to achieve the property of universal verifiability. However, proving the correctness of a shuffle without sacrificing unlinkability required a large amount of computation in the prior art. For example, SK95] adopted a cut-and-choose method to prove the correctness. Abe Ab99 took an approach to represent a shuffle using multiple pairwise permutations 1. In practical terms, however, neither scheme is efficient enough to handle a large number of ciphertexts, say on the order of 10,000.

This paper proposes a novel, efficient scheme for proving the correctness of a shuffle. We take a completely different approach than that of [SK95] and Ab99]. We represent a permutation by a matrix, and introduce two conditions which suffice to achieve a permutation matrix. We then present zero-knowledge proofs to prove the satisfiability of each condition. Moreover, these two proofs can be merged into one proof, resulting in a very efficient proof of a correct shuffle.

We also present here an analysis of the efficiency of our proof. Our proof requires roughly $18 n$ exponentiations to prove the correctness of a $n$-data shuffle,

\footnotetext{
${ }^{1}$ Another approach, based on a verifiable secret exponent multiplication is described in $\mathrm{Ne01}$.
} 
where as the protocol of Sako-Kilian SK95] required $642 n$ and that of Abe Ab99. required $22 n \log n$. Using the computation tools in [HAC], the total computation cost necessary in our proof can be reduced to an equivalent of $5 n$ exponentiations. The length of a proof will be only $2^{11} n$ bits in our protocol, opposed to $2^{18} n$ bits and $2^{14} n \log n$ bits required by Sako-Kilian and Abe, respectively.

Our paper is organized in the following way. In Section 2, we present the two conditions on a permutation matrix. In Section 4 we give zero-knowledge proofs for each of the two conditions, and discuss how these proofs are combined to achieve to prove the whole shuffle. In Section 5 we describe our protocol and in Section [6] we compare the efficiency of our protocol to prior work.

\section{Basic Idea}

\subsection{Shuffling}

Informally speaking, a shuffling is a procedure which on input of $n$ ciphertexts $\left(E_{1}, E_{2}, \ldots, E_{n}\right)$, outputs $n$ ciphertexts $\left(E_{1}^{\prime}, E_{2}^{\prime}, \ldots, E_{n}^{\prime}\right)$ where:

- there exists a permutation $\phi$ s.t $D\left(E_{i}^{\prime}\right)=D\left(E_{\phi^{-1}(i)}\right)$ for all $i$. Here, $D$ is a decryption algorithm for ciphertexts.

- Without the knowledge of $D$ or $\phi,\left(E_{1}, E_{2}, \ldots, E_{n}\right)$, and $\left(E_{1}^{\prime}, E_{2}^{\prime}, \ldots, E_{n}^{\prime}\right)$ reveal no information on the permutation $\phi$.

We consider the use of ElGamal cryptosystems, with public keys $(p, q, g, y)$ and secret key $X \in \mathbf{Z}_{\mathbf{q}}$ s.t. $y=g^{X} \bmod p$. 2

Given $n$ ciphertexts $\left\{E_{i}\right\}=\left\{\left(g_{i}, m_{i}\right)\right\}$, where all $\left\{g_{i}\right\}$ and $\left\{m_{i}\right\}$ have the order $q$, shuffled ciphertexts $\left\{E_{i}^{\prime}\right\}=\left\{\left(g_{i}^{\prime}, m_{i}^{\prime}\right)\right\}$ can be obtained by

$$
\begin{aligned}
& g_{i}^{\prime}=g^{r_{i}} \cdot g_{\phi^{-1}(i)} \bmod p \\
& m_{i}^{\prime}=y^{r_{i}} \cdot m_{\phi^{-1}(i)} \bmod p
\end{aligned}
$$

using randomly generated $\left\{r_{i}\right\}$.

\subsection{Permutation Matrix}

We define a matrix $\left(A_{i j}\right)$ to be a permutation matrix if it can be written as follow using some permutation function $\phi$.

$$
A_{i j}= \begin{cases}1 \bmod q & \text { if } \phi(i)=j \\ 0 \bmod q & \text { otherwise. }\end{cases}
$$

Using this permutation matrix, the equation (1) is equivalent to

$$
\left(g_{i}^{\prime}, m_{i}^{\prime}\right)=\left(g^{r_{i}} \prod_{j=1}^{n} g_{j}^{A_{j i}}, y^{r_{i}} \prod_{j=1}^{n} m_{j}^{A_{j i}}\right) \bmod p .
$$

In order to prove the correctness of the shuffle, we need to show the following two things.

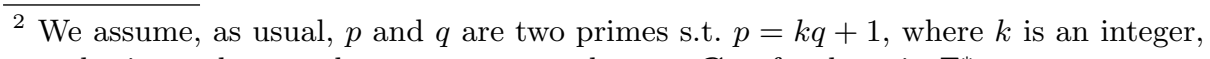
and $g$ is an element that generates a subgroup $\mathbf{G}_{\mathbf{q}}$ of order $q$ in $\mathbf{Z}_{\mathbf{p}}^{*}$. 
1. For each pair $\left\{\left(g_{i}^{\prime}, m_{i}^{\prime}\right)\right\}$, the same $r_{i}$ and $\left(A_{i j}\right)$ has been used.

2. $\left(A_{i j}\right)$ used is a permutation matrix.

The first property can be efficiently shown using a standard technique[Br93]. The contribution of this paper is to present a novel technique to prove the second property.

At first, we concentrate on proving the existence of a permutation matrix $\left(A_{i j}\right)$ and $\left\{r_{i}\right\}$ when given $\left\{g_{i}\right\}$ and $\left\{g_{i}^{\prime}\right\}$, s.t.

$$
g_{i}^{\prime}=g^{r_{i}} \prod_{j=1}^{n} g_{j}^{A_{j i}} \bmod p .
$$

We thus need to prove the existence of such a permutation matrix. We begin by looking at necessary conditions which suffices to achieve a permutation matrix. The following is the key observation used to construct the proposed protocol.

Theorem 1. A matrix $\left(A_{i j}\right)_{(i, j=1, \ldots, n)}$ is a permutation matrix if and only if, for all $i, j$, and $k$, both

$$
\begin{aligned}
\sum_{h=1}^{n} A_{h i} A_{h j} & = \begin{cases}1 \bmod q, & \text { if } i=j \\
0 \bmod q, & \text { if } i \neq j\end{cases} \\
\sum_{h=1}^{n} A_{h i} A_{h j} A_{h k} & = \begin{cases}1 \bmod q & \text { if } i=j=k \\
0 \bmod q & \text { if otherwise }\end{cases}
\end{aligned}
$$

hold.

Notation 1 For convenience, we define $\delta_{i j}$ and $\delta_{i j k}(i, j, k=1, \ldots, n)$ to be, respectively,

$$
\delta_{i j}=\left\{\begin{array}{ll}
1 & \text { if } i=j \\
0 & \text { if } i \neq j
\end{array} \quad \text { and } \quad \delta_{i j k}= \begin{cases}1 & \text { if } i=j=k \\
0 & \text { if otherwise }\end{cases}\right.
$$

Proof. We first show that there is exactly one non-zero element in each row vector of $\left(A_{i j}\right)$ and then, the same for each column vector.

Let $C_{i}$ be a $i$-th column vector of the matrix $\left(A_{i j}\right)_{(i, j=1, \ldots, n)}$. Then, from Equation (4), we see $\left(C_{i}, C_{j}\right)=\delta_{i j}$ where $(A, B)$ is inner product of vectors $A$ and $B$. This implies that $\operatorname{rank}\left(A_{i j}\right)=n$, that is, there is at least one non-zero element in each row and each column. Next we consider a vector $C_{i} \odot C_{j}(i \neq j)$ where the operator $\odot$ is defined as $\left(a_{1} \ldots a_{n}\right) \odot\left(b_{1} \ldots b_{n}\right)=\left(a_{1} b_{1} \ldots a_{n} b_{n}\right)$. Define a vector $\hat{C}=\sum_{l=0}^{n} \kappa_{l} C_{l}$ for an arbitrary $\kappa_{l}$. From the fact $\left(\hat{C}, C_{i} \odot C_{j}\right)=\sum_{l=1} \kappa_{l} \delta_{l i j}=0$ and linear combinations of $\left\{C_{l}\right\}$ generate the space $\mathbf{Z}_{\mathbf{q}}{ }^{n}$, we obtain $C_{i} \odot C_{j}=\mathbf{0}$. This means for any $h, i$ and $j$ s.t. $i \neq j$, either $A_{h i}=0$ or $A_{h j}=0$. Therefore, the number of non-zero elements in each row vector of $\left(A_{i j}\right)$ is at most 1 , and thus exactly 1.

From the above observations, the matrix $\left(A_{i j}\right)$ contains exactly $n$ non-zero elements. Since $C_{i} \neq \mathbf{0}$ for all $i$, the number of non-zero element in each column 
vector is also 1 . Thus, there is exactly one non-zero element in each row vector and each column vector of the matrix $\left(A_{i j}\right)$ if Equations (4) and (5) hold.

The unique non-zero element $e_{i}$ in $i-t h$ row must be $e_{i}^{2}=1 \bmod q$ from Equation (4) and $e_{i}^{3}=1 \bmod q$ from Equation (5). This leads to $e_{i}=1$ and that matrix $\left(A_{i j}\right)_{(i, j=1, \ldots, n)}$ is a permutation matrix over $\mathbf{Z}_{\mathbf{q}}$.

\subsection{Outline of Main Protocol}

Using Theorem 1 the main protocol can be constructed by the following proofs

Proof-1 a proof that given $\left\{g_{i}\right\}$ and $\left\{g_{i}^{\prime}\right\},\left\{g_{i}^{\prime}\right\}$ can be expressed as eq. (3) using integers $\left\{r_{i}\right\}$ and a matrix that satisfies the first condition.

Proof-2 a proof that given $\left\{g_{i}\right\}$ and $\left\{g_{i}^{\prime}\right\},\left\{g_{i}^{\prime}\right\}$ can be expressed as eq. (3) using integers $\left\{r_{i}\right\}$ and a matrix that satisfies the second condition.

Proof-3 a proof that integers $\left\{r_{i}\right\}$ and the matrix used in the above two proofs are identical.

Proof-4 For each pair $\left(g_{i}^{\prime}, m_{i}^{\prime}\right)$, the same $r_{i}$ and $\left\{A_{i j}\right\}$ has been used.

In the Section 4, we provide protocols for Proof-1 and Proof-2.

\section{Security of the Protocol}

We will prove that the main protocol is sound and zero-knowledge under computational assumption. More specifically, for the property of soundness, we can claim that if a verifier accepts the protocol, then either prover knows the permutation or he knows integers $\left\{a_{i}\right\}$ and $a$ satisfying $g^{a} \prod_{i=1}^{n} g_{i}{ }^{a_{i}}=1$ with overwhelming probability. For the zero-knowledge property, we can construct a simulator and claim that if there is a distinguisher who can distinguish between a real transcript from the protocol and an output from the simulator, then this distinguisher can be used to solve the decisional Diffie-Hellman problem. We note that to make a shuffle secret, we already assume the hardness of the decisional Diffie-Hellman problem.

In the course of reduction, we use the following arguments. First, we define the following set.

Definition 1. Define $R_{n}^{m}$ to be the set of tuples of $n \times m$ elements in $\mathbf{G}_{\mathbf{q}}$ :

$$
I=\left(x_{1}^{(1)}, \ldots, x_{1}^{(m)}, x_{2}^{(1)}, \ldots, x_{2}^{(m)}, \ldots, x_{n}^{(1)}, \ldots, x_{n}^{(m)}\right) .
$$

We then define the subset $D_{n}^{m}$ of $R_{n}^{m}$ to be the set of tuples I satisfying

$$
\log _{x_{1}^{(1)}} x_{1}^{(i)}=\log _{x_{j}^{(1)}} x_{j}^{(i)} \bmod p
$$

for all $i(i=2,3, . ., m)$ and $j(j=2, \ldots, n)$.

Definition 2. We define the problem of distinguishing instances uniformly chosen from $R_{n}^{m}$ and those from $D_{n}^{m}$ by $D D H_{n}^{m}$. 
Note that the decisional Diffie-Hellman problem can be denoted as $D D H_{2}^{2}$. We claim that for any $n$ and $m$ the difficulty of $D D H_{n}^{m}$ equals to the decisional Diffie-Hellman problem, by proving the following.

Lemma 1. For any $n(\geq 2)$ and $m(\geq 2)$, if $D D H_{n}^{m}$ is easy, then $D D H_{n}^{2}$ is easy.

Lemma 2. If for any $n(\geq 2), D D H_{n}^{2}$ is easy then the decisional Diffie-Hellman is easy.

Proofs for Lemma 1 and 2 are sketched in Appendix $\mathrm{A}$

\section{Proof-1 and Proof-2}

In this section, we give two proofs that will be the building blocks of the main protocol.

\subsection{Proving the First Condition (Proof-1)}

The following protocol proves that given $\left\{g_{i}\right\}$ and $\left\{g_{i}^{\prime}\right\}$, the prover knows $\left\{r_{i}\right\}$ and $\left\{A_{i j}\right\}$ s.t.

$$
\begin{aligned}
g_{i}^{\prime} & =g^{r_{i}} \prod_{j=1}^{n} g_{j}^{A_{j i}} \bmod p \\
\sum_{h=1}^{n} A_{h i} A_{h j} & =\delta_{i j} \bmod q .
\end{aligned}
$$

The main idea is to issue $s=\sum_{j=1}^{n} r_{j} c_{j}$ and $s_{i}=\sum_{j=1}^{n} A_{i j} c_{j}$ as a response to a challenge $\left\{c_{j}\right\}$ and let the verifier check

$$
\begin{aligned}
\sum_{i=1}^{n}{s_{i}}^{2} & =\sum_{j=1}^{n}{c_{j}}^{2} \bmod q \\
g^{s} \prod_{i=1}^{n} g_{i}{ }^{s_{i}} & =\prod_{j=1}^{n} g_{j}^{\prime c_{j}} \bmod p .
\end{aligned}
$$

However, this apparently leaks information on $A_{i j}$, so we need to add randomizers and commitments. By making the response $s=\sum_{j=1}^{n} r_{j} c_{j}+\alpha$ and $s_{i}=\sum_{j=1}^{n} A_{i j} c_{j}+\alpha_{i}$ using randomizers $\left\{\alpha_{i}\right\}$ and $\alpha$, a verifier needs to check the following equation:

$$
\sum_{i=1}^{n} s_{i}{ }^{2}=\sum_{j=1}^{n} c_{j}{ }^{2}+\sum_{j=1}^{n} B_{j} c_{j}+D \bmod q
$$

where $B_{j}$ and $D$ are quadratic polynomials of $\left\{A_{i j}\right\}$ and $\alpha_{i}$. Therefore these $B_{j}$ and $D$, together with $g^{\alpha} \prod_{i=1}^{n} g_{i}^{\alpha_{i}}$, will be also sent in advance to enable 
verification. We further add another randomizer $\sigma$ and modify the verification equation to be

$$
\sum_{i=1}^{n} s_{i}{ }^{2}+\sigma s=\sum_{j=1}^{n} c_{j}{ }^{2}+\sum_{j=1}^{n}\left(B_{j}+\sigma r_{j}\right) c_{j}+(D+\sigma \alpha) \bmod q
$$

In order to hide the actual value of $\sigma,\left\{B_{j}+\sigma r_{j}\right\}$ and $D+\sigma \alpha$, this verification is computed over exponents. The below gives a complete description of the Proof1.

\section{Proof-1}

Input:p, $q, g,\left\{g_{i}\right\},\left\{g_{i}^{\prime}\right\}$.

1. Prover $(\mathcal{P})$ generates random numbers $\sigma, \alpha,\left\{\alpha_{i}\right\}_{(i=1, \ldots, n)} \in_{R} \mathbf{Z}_{\mathbf{q}}$ and computes

$$
\begin{aligned}
w & =g^{\sigma} \bmod p \\
g^{\prime} & =g^{\alpha} \prod_{j=1}^{n} g_{j}{ }^{\alpha_{j}} \bmod p \\
\dot{w}_{i} & =g^{\sum_{j=1}^{n} 2 \alpha_{j} A_{j i}+\sigma r_{i}} \quad\left(=g^{B_{i}+\sigma r_{i}}\right) \bmod p \quad i=1, \ldots, n \\
\dot{w} & =g^{\sum_{j=1}^{n} \alpha_{j}{ }^{2}+\sigma \alpha} \quad\left(=g^{D+\sigma \alpha}\right) \bmod p
\end{aligned}
$$

and sends $w, g^{\prime},\left\{\dot{w}_{i}\right\}, \dot{w}(i=1, \ldots, n)$ to $\mathcal{V}$.

2. $\mathcal{V}$ sends back randomly chosen $\left\{c_{i}\right\}_{(i=1, \ldots, n)} \in_{R} \mathbf{Z}_{\mathbf{q}}$ as a challenge.

3. $\mathcal{P}$ computes $s=\sum_{j=1}^{n} r_{j} c_{j}+\alpha \bmod q$ and $s_{i}=\sum_{j=1}^{n} A_{i j} c_{j}+\alpha_{i} \bmod q(i=1, \ldots, n)$ and sends to $\mathcal{V}$.

4. $\mathcal{V}$ verifies the following:

$$
\begin{array}{r}
g^{s} \prod_{j=1}^{n} g_{j}{ }^{s_{j}}=g^{\prime} \prod_{j=1}^{n} g_{j}^{\prime c_{j}} \bmod p \\
w^{s} g^{\sum_{j=1}^{n}\left(s_{j}^{2}-c_{j}^{2}\right)}=\dot{w} \prod_{j=1}^{n} \dot{w}_{j}{ }^{c_{j}} \bmod p
\end{array}
$$

\section{Properties of Proof- 1}

Theorem 2. Proof-1 is complete. That is, if $\mathcal{P}$ knows $\left\{r_{i}\right\}$ and $\left\{A_{i j}\right\}$ satisfying the first condition, $\mathcal{V}$ always accepts.

Theorem 3. If $\mathcal{V}$ accepts Proof-1 with a non-negligible probability, then $\mathcal{P}$ either knows both $\left\{r_{i}\right\}$ and $\left\{A_{i j}\right\}$ satisfying the first condition, or can generate integers $\left\{a_{i}\right\}$ and a satisfying $g^{a} \prod_{i=1}^{n} g_{i}^{a_{i}}=1$ with overwhelming probability. 
A sketch of Proof: Theorem 3 can be proved from the following lemmas, proofs of which are sketched in Appendix B

Lemma 3. If $\mathcal{V}$ accepts Proof-1 with non-negligible probability, then $\mathcal{P}$ knows $\left\{A_{i j}\right\},\left\{r_{i}\right\},\left\{\alpha_{i}\right\}$, and $\alpha$ satisfying Equations (3) and (6).

Lemma 4. Assume $\mathcal{P}$ knows $\left\{A_{i j}\right\},\left\{r_{i}\right\},\left\{\alpha_{i}\right\}$, and $\alpha$ satisfying Equations (3) and (6). If $\mathcal{P}$ knows $\left\{s_{i}\right\}$ and $s$ which satisfy Equation (7), and either $s \neq$ $\sum_{j=1}^{n} r_{j} c_{j}+\alpha$ or $s_{i} \neq \sum_{j=1}^{n} A_{i j} c_{j}+\alpha_{i}$ for some $i$ hold, then $\mathcal{P}$ can generate non-trivial integers $\left\{a_{i}\right\}$ and a satisfying $g^{a} \prod_{i=1}^{n} g_{i}{ }^{a_{i}}=1$ with overwhelming probability.

Lemma 5. Assume $\mathcal{P}$ knows $\left\{A_{i j}\right\},\left\{r_{i}\right\},\left\{\alpha_{i}\right\}$, and $\alpha$ satisfying Equations (3) and (6). If Equations (7) and (8) hold with non-negligible probability, then either Equation (4) hold or $\mathcal{P}$ can generate non-trivial integers $\left\{a_{i}\right\}$ and a satisfying $g^{a} \prod_{i=1}^{n} g_{i}^{a_{i}}=1$ with overwhelming probability.

Theorem 4. We can construct a simulator of Proof-1 such that if there is a distinguisher who can distinguish between a real transcript from the protocol and an output from the simulator, then we can solve the decisional Diffie-Hellman problem.

A sketch of Proof: Given in Appendix B]

\subsection{Proving the Second Condition(Proof-2)}

Analogous to Proof-1, the proof for the fact the prover knows $\left\{r_{i}\right\}$ and $\left\{A_{i j}\right\}$ s.t.

$$
\begin{aligned}
g_{i}^{\prime} & =g^{r_{i}} \prod_{j} g_{j}^{A_{j i}} \bmod p \\
\sum_{h=1}^{n} A_{h i} A_{h j} A_{h k} & =\delta_{i j k} \bmod q
\end{aligned}
$$

for $\left\{g_{i}\right\}$ and $\left\{g_{i}^{\prime}\right\}$, is given as Proof-2.

\section{Proof-2}

Input: $p, q, g,\left\{g_{i}\right\},\left\{g_{i}^{\prime}\right\}$.

1. Prover $(\mathcal{P})$ generates random numbers $\rho, \tau, \alpha,\left\{\alpha_{i}\right\}, \lambda,\left\{\lambda_{i}\right\} \in_{R} \mathbf{Z}_{\mathbf{q}}$ $(i=1, \ldots, n)$ and computes

$$
\begin{aligned}
t & =g^{\tau}, v=g^{\rho}, u=g^{\lambda}, u_{i}=g^{\lambda_{i}} \bmod p \quad i=1, \ldots, n \\
g^{\prime} & =g^{\alpha} \prod_{j=1}^{n} g_{j}{ }^{\alpha_{j}} \bmod p \\
\dot{t}_{i} & =g^{\sum_{j=1}^{n} 3 \alpha_{j} A_{j i}+\tau \lambda_{i}} \bmod p \quad i=1, \ldots, n \\
\dot{v}_{i} & =g^{\sum_{j=1}^{n} 3 \alpha_{j}{ }^{2} A_{j i}+\rho r_{i}} \bmod p \quad i=1, \ldots, n \\
\dot{v} & =g^{\sum_{j=1}^{n} \alpha_{j}{ }^{3}+\tau \lambda+\rho \alpha} \bmod p
\end{aligned}
$$

and sends $t, v, u,\left\{u_{i}\right\}, g^{\prime},\left\{\dot{t}_{i}\right\},\left\{\dot{v}_{i}\right\}, \dot{v}(i=1, \ldots, n)$, to $\mathcal{V}$. 
2. $\mathcal{V}$ sends back randomly chosen $\left\{c_{i}\right\}_{(i=1, \ldots, n)} \in_{R} \mathbf{Z}_{\mathbf{q}}$ as challenge.

3. $\mathcal{P}$ computes $s=\sum_{j=1}^{n} r_{j} c_{j}+\alpha \bmod q, s_{i}=\sum_{j=1}^{n} A_{i j} c_{j}+\alpha_{i} \bmod q(i=$ $1, \ldots, n)$, and $\lambda^{\prime}=\sum_{j=1}^{n} \lambda_{j} c_{j}^{2}+\lambda \bmod q$ and sends to $\mathcal{V}$.

4. $\mathcal{V}$ verifies the following:

$$
\begin{aligned}
& g^{s} \prod_{j=1}^{n} g_{j}^{s_{j}}=g^{\prime} \prod_{j=1}^{n} g_{j}^{c^{c_{j}} \bmod p} \\
& g^{\lambda^{\prime}}=u \prod_{j=1}^{n} u_{j}{ }^{c_{j}^{2}} \bmod p \\
& t^{\lambda^{\prime}} v^{s} g^{\sum_{j=1}^{n}\left(s_{j}^{3}-c_{j}^{3}\right)}=\dot{v} \prod_{j=1}^{n} \dot{v}_{j}{ }^{c_{j}} \dot{t}_{j} c_{j}^{2} \bmod p
\end{aligned}
$$

\section{Properties of Proof-2}

We claim the following properties of Proof-2, which can be proved analogously to that of Proof-1.

Theorem 5. Proof-2 is complete. That is, if $\mathcal{P}$ knows $\left\{r_{i}\right\}$ and $\left\{A_{i j}\right\}$ satisfying the second condition, $\mathcal{V}$ always accepts.

Theorem 6. If $\mathcal{V}$ accepts Proof-2 with a non-negligible probability, then $\mathcal{P}$ either knows both $\left\{r_{i}\right\}$ and $\left\{A_{i j}\right\}$ satisfying the second condition, or can generate integers $\left\{a_{i}\right\}$ and a satisfying $g^{a} \prod_{i=1}^{n} g_{i}{ }^{a_{i}}=1$ with overwhelming probability.

Theorem 7. We can construct a simulator of Proof-2 such that if there is a distinguisher who can distinguish between a real transcript from the protocol and an output from the simulator, then we can solve the decisional Diffie-Hellman problem.

\subsection{Constructing the Main Protocol}

In this subsection, we explain how our main protocol is constructed using these proof-1 and Proof-2. It should be noted, that these proofs did not have the ordinary soundness property. That is, a prover knowing integers satisfying $g^{a} \prod_{i=1}^{n} g_{i}^{a_{i}}=1$ can deceive verifiers as if he had shuffled correctly. Since $\left\{g_{i}\right\}$ is originally chosen by those who encrypted the messages, there is no control to assure that the prover does not know the relations among them. Therefore, we fix a set of basis $\left\{\tilde{g}, \tilde{g}_{1}, \ldots \tilde{g}_{n}\right\}$ independent from the input ciphertexts, in a way we can assure the relations among the basis unknown. In fact, under Discrete Logarithm Assumption, we can make it computationally infeasible to obtain such $\left\{a_{i}\right\}$ and $a$ if we generate $\left\{\tilde{g}, \tilde{g}_{1}, \ldots \tilde{g}_{n}\right\}$ randomly Br93. This way it also suffices the requirement that the verifier should not know $\log _{g} \tilde{g}$ for zeroknowledge property.

We require the prover to perform the same permutation on the set of fixed basis as he did on the input ciphertexts. The prover proves that the permutation 
on the fixed basis $\left\{\tilde{g}, \tilde{g}_{1}, \ldots \tilde{g}_{n}\right\}$ is indeed a permutation, and that he indeed applied the same permutation to the input ciphertext.

Using the above methodology, we need not provide Proof-3 described in the Subsection 2.3 If a prover knows two different representations of an element using $\left\{\tilde{g}, \tilde{g}_{1}, \ldots \tilde{g}_{n}\right\}$, it means that he knows the relations among the base $\left\{\tilde{g}, \tilde{g}_{1}, \ldots \tilde{g}_{n}\right\}$ which is against the assumption. Proof-4 is achieved using the standard techniques described in Br93. Therefore we are now equipped with building blocks to prove the correctness of a shuffle.

\section{The Main Protocol}

In the previous subsection we illustrated our protocol as a combination of proof$\mathbf{1}$ and proof-2, mainly for comprehensiveness. The proofs can be executed in parallel, resulting in a three-round protocol with reduced communication complexity.

\section{Main Protocol}

Input:p, $q, g, y, \tilde{g},\left\{\tilde{g}_{i}\right\},\left\{\left(g_{i}, m_{i}\right)\right\},\left\{\left(g_{i}^{\prime}, m_{i}^{\prime}\right)\right\}$.

1. Prover $(\mathcal{P})$ generates the following random numbers: $\sigma, \rho, \tau, \alpha, \alpha_{i}, \lambda, \lambda_{i} \in_{R} \mathbf{Z}_{q}(i=1, \ldots, n)$

2. $\mathcal{P}$ computes the following:

$$
\begin{aligned}
t & =g^{\tau}, v=g^{\rho}, w=g^{\sigma}, u=g^{\lambda}, u_{i}=g^{\lambda_{i}} \bmod p \quad i=1, \ldots, n \\
\tilde{g}_{i}^{\prime} & =\tilde{g}^{r_{i}} \prod_{j=1}^{n} \tilde{g}_{j}^{A_{j i}} \bmod p \quad i=1, \ldots, n \\
\tilde{g}^{\prime} & =\tilde{g}^{\alpha} \prod_{j=1}^{n} \tilde{g}_{j}^{\alpha_{j}} \bmod p \\
g^{\prime} & =g^{\alpha} \prod_{j=1}^{n} g_{j}{ }^{\alpha_{j}} \bmod p \\
m^{\prime} & =y^{\alpha} \prod_{j=1}^{n} m_{j}{ }^{\alpha_{j}} \bmod p \\
\dot{t}_{i} & =g^{\sum_{j=1}^{n} 3 \alpha_{j} A_{j i}+\tau \lambda_{i}} \bmod p \quad i=1, \ldots, n \\
\dot{v}_{i} & =g^{\sum_{j=1}^{n} 3 \alpha_{j}{ }^{2} A_{j i}+\rho r_{i}} \bmod p \quad i=1, \ldots, n \\
\dot{v} & =g^{\sum_{j=1}^{n} \alpha_{j}{ }^{3}+\tau \lambda+\rho \alpha} \bmod p \\
\dot{w_{i}} & =g^{\sum_{j=1}^{n} 2 \alpha_{j} A_{j i}+\sigma r_{i}} \bmod p \quad i=1, \ldots, n \\
\dot{w} & =g^{\sum_{j=1}^{n} \alpha_{j}{ }^{2}+\sigma \alpha} \bmod p .
\end{aligned}
$$

3. $\mathcal{P}$ sends the following to the verifier $\mathcal{V}$ :

$t, v, w, u,\left\{u_{i}\right\},\left\{\tilde{g}_{i}^{\prime}\right\}, \tilde{g}^{\prime}, g^{\prime}, m^{\prime},\left\{\dot{t}_{i}\right\},\left\{\dot{v}_{i}\right\}, \dot{v},\left\{\dot{w}_{i}\right\}, \dot{w}(i=1, \ldots, n)$. 
4. $\mathcal{V}$ sends back randomly chosen $\left\{c_{i}\right\}_{(i=1, \ldots, n)} \in_{R} \mathbf{Z}_{\mathbf{q}}$ as a challenge.

5. $\mathcal{P}$ computes the following and sends them to $\mathcal{V}$.

$$
\begin{aligned}
s & =\sum_{j=1}^{n} r_{j} c_{j}+\alpha, \quad s_{i}=\sum_{j=1}^{n} A_{i j} c_{j}+\alpha_{i} \bmod q \quad i=1, \ldots, n \\
\lambda^{\prime} & =\sum_{j=1}^{n} \lambda_{j} c_{j}^{2}+\lambda \bmod q
\end{aligned}
$$

6. $\mathcal{V}$ verifies the following:

$$
\begin{aligned}
& \tilde{g}^{s} \prod_{j=1}^{n} \tilde{g}_{j}^{s_{j}}=\tilde{g}^{\prime} \prod_{j=1}^{n} \tilde{g}_{j}^{\prime c_{j}} \bmod p \\
& g^{s} \prod_{j=1}^{n} g_{j}{ }^{s_{j}}=g^{\prime} \prod_{j=1}^{n} g_{j}^{\prime c_{j}} \bmod p \\
& y^{s} \prod_{j=1}^{n} m_{j}{ }^{s_{j}}=m^{\prime} \prod_{j=1}^{n} m_{j}^{\prime c_{j}} \bmod p \\
& g^{\lambda^{\prime}}=u \prod_{j=1}^{n} u_{j}{ }^{c_{j}^{2}} \bmod p \\
& t^{\lambda^{\prime}} v^{s} g^{\sum_{j=1}^{n}\left(s_{j}^{3}-c_{j}^{3}\right)}=\dot{v} \prod_{j=1}^{n} \dot{v}_{j}{ }^{c_{j}} \dot{t}_{j} c_{j}^{2} \bmod p \\
& w^{s} g^{\sum_{j=1}^{n}\left(s_{j}^{2}-c_{j}^{2}\right)}=\dot{w} \prod_{j=1}^{n} \dot{w}_{j}^{c_{j}} \bmod p
\end{aligned}
$$

Theorem 8. Main Protocol is complete. That is, if $\mathcal{P}$ knows $\left\{r_{i}\right\}$ and $\left\{A_{i j}\right\}$ satisfying the both conditions of Theorem $1 \mathcal{V}$ always accepts.

Theorem 9. If $\mathcal{V}$ accepts Main Protocol with a non-negligible probability, then $\mathcal{P}$ knows $\left\{r_{i}\right\}$ and permutation matrix $\left(A_{i j}\right)$ satisfying Equations (2), or can generate non-trivial integers $\left\{a_{i}\right\}$ and a satisfying $\tilde{g}^{a} \prod_{i=1}^{n} \tilde{g}_{i}^{a_{i}}=1$ with overwhelming probability.

Theorem 10. We can construct a simulator of Main Protocol such that if there is a distinguisher who can distinguish between a real transcript from the protocol and an output from the simulator, then we can solve the decisional DiffieHellman problem.

Proofs for Theorem 9 and 10 are sketched in Appendix C 


\section{Discussions}

In this section, we compare the efficiency of the proposed protocol described in Section 5 to the SK95 protocol in SK95 and MiP-2 protocol in Ab99. To enable a fair comparison, we assume the security parameter of SK95] to be 160 and lengths of $p$ and $q$ to be 1024 and 160 respectively.

We first compare them by the number of exponentiations used in each protocol, in the case of shuffling $n$ ciphertexts. These are $22(n \log n-n+1)$ for Abe's protocol, $642 n$ for the SK95 protocol, and $18 n+18$ for the proposed protocol. If we adopt computation tools described in [HAC], such as the simultaneous multiple exponentiation algorithm and the fixed-base comb method, the number of exponentiations can be heuristically reduced to $11.2(n \log n-n+1), 64 n$, and $4.84 n+4.5$, respectively. The total number of bits needing to be transfered during the protocols is $13,248(n \log n-n+1), 353,280 n$, and $5,280 n+13,792$. The rounded-up numbers are shown in Table [1]

Table 1. Comparison of three protocols

\begin{tabular}{||c||c|c|c|}
\hline & Abe $(\mathrm{MiP}-2)$ & SK95 & This Paper \\
\hline No. exponentiations & $22 n \log n$ & $642 n$ & $18 n$ \\
\hline (heuristically adjusted) & $11 n \log n$ & $64 n$ & $5 n$ \\
\hline No. communication bits & $2^{14} n \log n$ & $2^{18} n$ & $2^{11} n$ \\
\hline
\end{tabular}

\section{Conclusion}

In this paper, we presented a novel method to prove the correctness of a shuffle, and demonstrated its efficiency. The proposed method requires only $18 n$ exponentiations for shuffling $n$ ciphertexts, where as previous methods required 35 times more, or required a higher order, $O(n \log n)$.

The proposed protocol can be used to build an efficient, universally verifiable voting system where the number of voters can scale up to the order of 10,000 .

Acknowledgments. The authors would like to thank Tatsuaki Okamoto, Masayuki Abe, and Satoshi Obana for many helpful discussions.

\section{References}

[Ab99] M. Abe, Mix-Networks on Permutation Networks, Asiacrypt '99, LNCS 1716, 258-273 (1999)

[Br93] S. Brands, An Efficient Off-line Electronic Cash System Based On The Representation Problem, CWI Technical Report CS-R9323, (1993)

[Ch81] D. Chaum, Untraceable Electronic Mail, Return Addresses, and Digital Pseudonyms, Communications of the ACM, Vol.24, No.2 84-88 (1981)

[CDS94] R. Cramer, I. Damgård and B. Schoenmakers, Proofs of Partial Knowledge and Simplified Design of Witness Hiding Protocols, Crypto '94, LNCS 839, 174-187 (1994) 
[HAC] A. Menezes, C. van Oorschot and S. Vanstone, Handbook of Applied Cryptography, CRC Press, 617-619

[Ne01] C.A. Neff, Verifiable, Secret Shuffles of ElGamal Encrypted Data, Initial version circulated Mar. 2000, current version submitted to ACMCCS 01

[OKST97] W. Ogata, K. Kurosawa, K. Sako and K. Takatani, Fault tolerant anonymous channel, 1st International Conference on Information and Communications Security (ICICS), LNCS 1334, 440-444 (1997)

[SK95] K. Sako and J. Kilian, Receipt-free mix-type voting scheme - A practical solution to the implementation of voting booth, Eurocrypt 95, LNCS 921, 393-403 (1995)

\section{A $\quad D D H_{n}^{m}$ and DDH}

Lemma 1. For any $m(\geq 2)$ and $n(\geq 2)$, if $D D H_{n}^{m}$ is easy, then $D D H_{n}^{2}$ is easy. Proof. We claim that if $D D H_{n}^{m}$ is easy, then either $D D H_{n}^{m-1}$ is easy or $D D H_{n}^{2}$ is easy. By induction we can prove the correctness of the lemma.

In order to prove the claim, we define the subset $M_{n}^{m}$ of $R_{n}^{m}$ to be the set of tuples

$$
I=\left(x_{1}^{(1)}, \ldots, x_{1}^{(m)}, x_{2}^{(1)}, \ldots, x_{2}^{(m)}, \ldots, x_{n}^{(1)}, \ldots, x_{n}^{(m)}\right)
$$

satisfying

$$
\log _{x_{1}^{(1)}} x_{1}^{(i)}=\log _{x_{j}^{(1)}} x_{j}^{(i)} \bmod p
$$

for all $i(i=2,3, . ., m-1)$ and $j(j=2, \ldots, n)$, but whether or not

$$
\log _{x_{1}^{(1)}} x_{1}^{(m)}=\log _{x_{j}^{(1)}} x_{j}^{(m)} \bmod q
$$

holds for all $j(j=2, \ldots, n)$ is arbitrary. Therefore, the set $D_{n}^{m}$ is a subset of $M_{n}^{m}$.

It is clear that if $D D H_{n}^{m}$ is easy, then we can either distinguish between the instances chosen uniformly from $R_{n}^{m}$ and $M_{n}^{m}$ or the instances chosen uniformly from $M_{n}^{m}$ and $D_{n}^{m}$. In the former case, it means $D D H_{n}^{m-1}$ is easy. We claim in the following that in the latter case $D D H_{n}^{2}$ is easy.

Assume $M_{n}^{m}$ and $D_{n}^{m}$ are distinguishable. For any $I_{n}^{2} \in R_{n}^{2}$ s.t.

$$
I_{n}^{2}=\left(x_{1}^{(1)}, x_{1}^{(2)}, x_{2}^{(1)}, x_{2}^{(2)}, \ldots, x_{n}^{(1)}, x_{n}^{(2)}\right)
$$

we transform it to $I_{n}^{m} \in R_{n}^{m}$

$$
I_{n}^{m}=\left(x_{1}^{\prime(1)}, \ldots, x_{1}^{\prime(m)}, x_{2}^{\prime(1)}, \ldots, x_{2}^{\prime(m)}, \ldots, x_{n}^{\prime(1)}, \ldots, x_{n}^{\prime(m)}\right)
$$

where

$$
x^{\prime}{ }_{j}^{(i)}= \begin{cases}x_{j}^{(1)} & j=1, \ldots, n(\text { if } i=1) \\ \left(x_{j}^{(1)}\right)^{z_{i}} \bmod p j & =1, \ldots, n(\text { if } 2 \leq i \leq m-1) \\ x_{j}^{(2)} & j=1, \ldots, n(\text { if } i=m)\end{cases}
$$

with randomly chosen $\left\{z_{i}\right\}_{(i=2, \ldots, m-1)}$ in $\mathbf{Z}_{\mathbf{q}}$.

If $I_{n}^{2}$ is chosen uniformly from $D_{n}^{2}$, then $I_{n}^{m}$ is distributed uniformly in $D_{n}^{m}$, and if $I_{n}^{2}$ is chosen uniformly from $R_{n}^{2}$, then $I_{n}^{m}$ is distributed uniformly in $M_{n}^{m}$. Therefore if $D_{n}^{m}$ and $M_{n}^{m}$ is distinguishable, then we can solve $D D H_{n}^{2}$. 
Lemma 2. If $D D H_{n}^{2}(n \geq 2)$ is easy then the decisional Diffie-Hellman problem $\left(D D H_{2}^{2}\right)$ is easy.

Proof. For any $I_{2}^{2}=\left(x_{1}^{(1)}, x_{1}^{(2)}, x_{2}^{(1)}, x_{2}^{(2)}\right) \in R_{2}^{2}$, we transform it to $I_{n}^{2} \in R_{n}^{2}$

$$
I_{n}^{2}=\left(x_{1}^{\prime(1)}, x_{1}^{\prime(2)}, x_{2}^{\prime(1)}, x_{2}^{\prime(2)}, \ldots, x_{n}^{\prime(1)}, x_{n}^{\prime(2)}\right)
$$

where

$$
\begin{aligned}
& x_{1}^{\prime(1)}=x_{1}^{(1)}, x_{1}^{(2)}=x_{1}^{(2)}, x_{2}^{\prime(1)}=x_{2}^{(1)}, x_{2}^{(2)}=x_{2}^{(2)} \\
& x^{\prime(1)}=\left(x_{1}^{(1)}\right)^{z_{j}} \cdot\left(x_{2}^{(1)}\right)^{w_{j}}, \quad x_{j}^{\prime(2)}=\left(x_{1}^{(2)}\right)^{z_{j}} \cdot\left(x_{2}^{(2)}\right)^{w_{j}} \bmod p \quad j=3, \ldots, n
\end{aligned}
$$

with randomly chosen $\left\{z_{j}\right\}$ and $\left\{w_{j}\right\}(j=3, \ldots, n)$ in $\mathbf{Z}_{\mathbf{q}}$.

If $I_{2}^{2}$ is chosen uniformly from $D_{2}^{2}$, then $I_{n}^{2}$ is distributed uniformly in $D_{n}^{2}$, and if $I_{2}^{2}$ is chosen uniformly from $R_{2}^{2}$, then $I_{n}^{2}$ is distributed uniformly in $R_{n}^{2}$. Therefore if $D D H_{n}^{2}$ is easy, then so is $D D H_{2}^{2}$.

\section{B Properties of Proof-1}

In this section, we sketch the proofs of the following theorems.

Theorem 3 (soundness). If $\mathcal{V}$ accepts Proof-1 with a non-negligible probability, then $\mathcal{P}$ either knows both $\left\{r_{i}\right\}$ and $\left\{A_{i j}\right\}$ satisfying the first condition, or can generate integers $\left\{a_{i}\right\}$ and a satisfying $g^{a} \prod_{i=1}^{n} g_{i}{ }^{a_{i}}=1$ with overwhelming probability.

Theorem 4 (zero-knowledge). We can construct a simulator of Proof-1 such that if there is a distinguisher who can distinguish between a real transcript from the protocol and an output from the simulator, then we can solve the decisional Diffie-Hellman problem.

\section{B.1 Soundness}

It is clear that Theorem 3 holds if Lemmas 3, 4 and 5] hold. We therefore prove the lemmas.

Lemma 3. If $\mathcal{V}$ accepts Proof-1 with non-negligible probability, then $\mathcal{P}$ knows $\left\{A_{i j}\right\},\left\{r_{i}\right\},\left\{\alpha_{i}\right\}$, and $\alpha$ satisfying Equations (3) and (6).

$A$ sketch of Proof: Define $\mathcal{C}_{p}$ as the space which is spanned by the vector $\left(1, c_{1}, c_{2}, \ldots, c_{n}\right)$ made of the challenges to which $\mathcal{P}$ can compute responses $s,\left\{s_{i}\right\}_{(i=1, \ldots, n)}$ such that Equation (17) holds. If the $\operatorname{dim}\left(\mathcal{C}_{p}\right)=n+1, \mathcal{P}$ can choose $n+1$ challenges which are linearly independent and obtain $\left\{A_{i j}\right\}_{(i, j=1, \ldots, n)}$, $\left\{r_{i}\right\}_{(i=1, \ldots, n)},\left\{\alpha_{i}\right\}_{(i=1, \ldots, n)}$, and $\alpha$ which satisfies the relation:

$$
s=\sum_{j=1}^{n} r_{j} c_{j}+\alpha, \quad s_{i}=\sum_{j=1}^{n} A_{i j} c_{j}+\alpha_{i} \bmod q \quad i=1, \ldots, n
$$

Such $\left\{A_{i j}\right\},\left\{r_{i}\right\},\left\{\alpha_{i}\right\}$, and $\alpha$ satisfies Equations (3) and (6) . If, $\operatorname{dim}\left(\mathcal{C}_{p}\right)<n+1$. The probability that $\mathcal{V}$ generates a challenge in $\mathcal{C}_{p}$ is at most $q^{n-1} / q^{n}=1 / q$, which is negligible. 
Lemma 4. Assume $\mathcal{P}$ knows $\left\{A_{i j}\right\},\left\{r_{i}\right\},\left\{\alpha_{i}\right\}$, and $\alpha$ satisfying Equations (3) and (6). If $\mathcal{P}$ knows $\left\{s_{i}\right\}$ and $s$ which satisfy Equation (7), and either $s \neq$ $\sum_{j=1}^{n} r_{j} c_{j}+\alpha$ or $s_{i} \neq \sum_{j=1}^{n} A_{i j} c_{j}+\alpha_{i}$ for some $i$ hold, then $\mathcal{P}$ can generate non-trivial integers $\left\{a_{i}\right\}$ and a satisfying $g^{a} \prod_{i=1}^{n} g_{i}{ }^{a_{i}}=1$ with overwhelming probability.

Proof. The following gives a non-trivial representation of 1 using $g,\left\{g_{i}\right\}$.

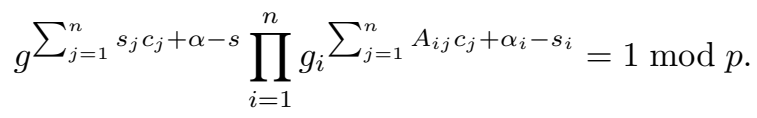

Lemma 5. Assume $\mathcal{P}$ knows $\left\{A_{i j}\right\},\left\{r_{i}\right\},\left\{\alpha_{i}\right\}$, and $\alpha$ satisfying Equations (3) and (6). If Equations (7) and (8) hold with non-negligible probability, then either Equation (4) hold or $\mathcal{P}$ can generate non-trivial integers $\left\{a_{i}\right\}$ and a satisfying $g^{a} \prod_{i=1}^{n} g_{i}^{a_{i}}=1$ with overwhelming probability.

A sketch of Proof: From Lemma 4 If Equation (7) holds, then either

$$
\left\{\begin{aligned}
s & =\sum_{j=1}^{n} r_{j} c_{j}+\alpha \bmod q \\
s_{i} & =\sum_{j=1}^{n} A_{i j} c_{j}+\alpha_{i} \bmod q \quad i=1, \ldots, n
\end{aligned}\right.
$$

holds or $\mathcal{P}$ can generate non-trivial integers $\left\{a_{i}\right\}$ and $a$ satisfying $g^{a} \prod_{i=1}^{n} g_{i}{ }^{a_{i}}=1$ with overwhelming probability. We concentrate on the former case. If Equation (8) holds, then

$$
\begin{aligned}
& \sum_{i=1}^{n} \sum_{j=1}^{n}\left(\sum_{h=1}^{n} A_{h i} A_{h j}-\delta_{i j}\right) c_{i} c_{j}+\sum_{i=1}^{n}\left\{\left(\sum_{j=1}^{n} 2 \alpha_{j} A_{j i}+\sigma r_{i}\right)-\psi_{i}\right\} c_{i} \\
& +\left\{\left(\sum_{j=1}^{n} \alpha_{j}^{2}+\sigma \alpha\right)-\psi\right\}=0 \quad \bmod q
\end{aligned}
$$

where $\psi_{i}=\sum_{j=1}^{n} 2 \alpha_{j} A_{j i}+\sigma r_{i}, \psi=\sum_{j=1}^{n} \alpha_{j}{ }^{2}+\sigma \alpha \bmod q$. If Equation (4) does not hold for some $i$ and $j$, then the probability that Equation (8) holds is negligible.

\section{B.2 Zero-Knowledge}

A sketch of Proof: We first give a construction of the simulator. We then prove that if there exists such a distinguisher then we can solve $D D H_{n+1}^{2}$. From Lemma 2 it means it is equivalent to solving the decisional Diffie-Hellman problem. 


\section{The Construction of the Simulator}

We will construct the simulator $\mathcal{S}$ of the Proof-1 with the input $p, q, g,\left\{g_{i}\right\}$, $\left\{g_{i}^{\prime}\right\}$ as follows.

The simulator $\mathcal{S}$ first generates $s,\left\{s_{i}\right\},\left\{c_{i}\right\} \in_{R} \mathbf{Z}_{\mathbf{q}}, w,\left\{\dot{w}_{i}\right\} \in_{R} \mathbf{G}_{\mathbf{q}}$ randomly. Then it computes $g^{\prime}, \dot{w}$ as the following.

$$
\begin{aligned}
& g^{\prime}=g^{s} \prod_{j=1}^{n} g_{j}^{s_{j}} g_{j}^{\prime-c_{j}} \bmod p \\
& \dot{w}=w^{s} g^{\sum_{j=1}^{n}\left(s_{j}^{2}-c_{j}^{2}\right)} \prod_{j=1}^{n} \dot{w}_{j}^{-c_{j}} \bmod p
\end{aligned}
$$

The output of $\mathcal{S}$ is $\left(w, g^{\prime},\left\{\dot{w}_{i}\right\}, \dot{w},\left\{c_{i}\right\}, s,\left\{s_{i}\right\}\right)$

\section{A Distinguisher of $D_{n+1}^{2}$ and $R_{n+1}^{2}$}

We will then construct a distinguisher $\mathcal{D}^{\prime}$ who can distinguish between the uniform instances of $D_{n+1}^{2}$ and $R_{n+1}^{2}$ if $\mathcal{S}$ can not simulate the Proof-1.

Let's say the instance $I=\left(x_{1}^{(1)}, x_{1}^{(2)}, \ldots, x_{n+1}^{(1)}, x_{n+1}^{(2)}\right)$ was chosen uniformly from either $D_{n+1}^{2}$ or $R_{n+1}^{2}$. Then this distinguisher will first generate $g_{1}, g_{2}, . . g_{n}$ as the constants used in Proof-1 and let $g=x_{1}^{(1)}$.

It will then generate a random permutation matrix $\left(A_{j i}\right)$ and compute

$$
g_{i}^{\prime}=x_{i+1}^{(1)} \prod_{j=1}^{n} g_{j}^{A_{j i}} \bmod p . \quad(i=1, \ldots, n)
$$

We note that $\left\{g_{i}^{\prime}\right\}$ gives a random permutation of $\left\{g_{i}\right\}$.

Based on $g,\left\{g_{i}\right\},\left\{g_{i}^{\prime}\right\}$, the distinguisher $\mathcal{D}^{\prime}$ is going to act as a simulator $\mathcal{S}^{\prime}$ which simulates the simulator $\mathcal{S}$. More specifically, the simulator $\mathcal{S}^{\prime}$ randomly generates $s,\left\{s_{i}\right\},\left\{c_{i}\right\} \in_{R} \mathbf{Z}_{\mathbf{q}}$ and computes

$$
\begin{aligned}
w & =x_{1}^{(2)} \\
g^{\prime} & =g^{s} \prod_{j=1}^{n} g_{j}^{s_{j}} g_{j}^{\prime}-c_{j} \bmod p \\
\alpha_{j} & =s_{j}-\sum_{k=1}^{n} A_{j k} c_{k} \bmod q \quad j=1, \ldots, n \\
\dot{w}_{i} & =x_{i+1}^{(2)} \prod_{j=1}^{n} g^{2 \alpha_{j} A_{j i}} \bmod p \quad i=1, \ldots, n \\
\dot{w} & =w^{s} g^{\sum_{j=1}^{n}\left(s_{j}^{2}-c_{j}^{2}\right)} \prod_{j=1}^{n} \dot{w}_{j}^{-c_{j}} \bmod p .
\end{aligned}
$$

The simulator $\mathcal{S}^{\prime}$ outputs

$$
w, g^{\prime},\left\{\dot{w}_{i}\right\}, \dot{w},\left\{c_{i}\right\}, s,\left\{s_{i}\right\} \cdot(i=1, \ldots, n)
$$


Lemma 6. Simulator $\mathcal{S}^{\prime}$ perfectly simulates Proof-1 when $I \in_{R} D_{n+1}^{2}$.

Sketch: We let

$$
\log _{x_{1}^{(1)}} x_{i+1}^{(1)}=r_{i}, \quad \log _{x_{1}^{(1)}} x_{1}^{(2)}=\sigma .
$$

Then it is clear that by randomly choosing $\left\{s_{i}\right\}$ and $s$, it gives the same distribution of the output as when $\left\{\alpha_{i}\right\}$ and $\alpha$ were first chosen randomly, and verifier honestly chooses random challenge $\left\{c_{i}\right\}$.

Lemma 7. Simulator $\mathcal{S}^{\prime}$ perfectly simulates $\mathcal{S}$ when $I \in_{R} R_{n+1}^{2}$.

Since $\left\{x_{i}^{(2)}\right\}_{(i=1, \ldots, n+1)}$ are randomly chosen, it gives the same distribution when $\dot{w}_{i}$ and $w$ are randomly chosen.

Therefore, if there exists a distinguisher $\mathcal{D}$ that distinguishes the output of the simulator $\mathcal{S}$ and a real transcript of Proof-1, then this distinguisher can be used to solve $D D H_{n+1}^{2}$.

\section{Properties of the Main Protocol}

In this section, we discuss the properties of the main protocol. The completeness property is clear. We provide proofs for the soundness and the zero-knowledge property.

\section{C.1 Soundness}

Theorem 9. If $\mathcal{V}$ accepts Main Protocol with a non-negligible probability, then $\mathcal{P}$ knows $\left\{r_{i}\right\}$ and permutation matrix $\left(A_{i j}\right)$ satisfying Equations (2), or can generate non-trivial integers $\left\{a_{i}\right\}$ and a satisfying $\tilde{g}^{a} \prod_{i=1}^{n} \tilde{g}_{i}^{a_{i}}=1$ with overwhelming probability.

A sketch of Proof:

We can show $\mathcal{P}$ 's knowledge of $\left\{A_{i j}\right\},\left\{r_{i}\right\},\left\{\alpha_{i}\right\}$, and $\alpha$ satisfying Equations (9) and (10) from the satisfiability of Equation (11), similar to Lemma 3 From the satisfiability of Equations (11) and (16), and additionally that of Equations (14) and (15), we can prove that the $\left\{A_{i j}\right\}$ satisfies the both conditions of Theorem 1, in a similar manner as proving Lemma 5 Thus Theorem 1 ensures that $\left(A_{i j}\right)$ is a permutation matrix. The following lemma ensures that the same permutation matrix was applied to both $\left\{g_{i}\right\}$ and $\left\{m_{i}\right\}$ to achieve $\left\{g_{i}^{\prime}\right\}$ and $\left\{m_{i}^{\prime}\right\}$, yielding the correctness of the shuffle.

Lemma 8. Assume $\mathcal{P}$ knows $\left\{A_{i j}\right\},\left\{r_{i}\right\},\left\{\alpha_{i}\right\}$, and $\alpha$ satisfying Equations (9) and (10), and $\left\{s_{i}\right\}$ and $s$ satisfying Equation (11). If Equations (12) and (13) hold with non-negligible probability, then either the relationships 


$$
\left\{\begin{array}{l}
g^{\prime}=g^{\alpha} \prod_{j=1}^{n} g_{j}{ }^{\alpha_{j}} \bmod p \\
g_{i}^{\prime}=g^{r_{i}} \prod_{j=1}^{n} g_{j}{ }^{A_{j i}} \bmod p \quad i=1, \ldots, n \\
m^{\prime}=y^{\alpha} \prod_{j=1}^{n} m_{j}{ }^{\alpha_{j}} \bmod p \\
m_{i}^{\prime}=y^{r_{i}} \prod_{j=1}^{n} m_{j}{ }^{A_{j i}} \bmod p \quad i=1, \ldots, n
\end{array}\right.
$$

hold or $\mathcal{P}$ can generate nontrivial integers $\left\{a_{i}\right\}$ and a satisfying $\tilde{g}^{a} \prod_{i=1}^{n} \tilde{g}_{i}{ }^{a_{i}}=1$ with overwhelming probability.

A sketch of Proof: Similarly to Lemma 4 we can ensure that

$$
\left\{\begin{aligned}
s & =\sum_{j=1}^{n} r_{j} c_{j}+\alpha \bmod q \\
s_{i} & =\sum_{j=1}^{n} A_{i j} c_{j}+\alpha_{i} \bmod q \quad i=1, \ldots, n
\end{aligned}\right.
$$

hold from the satisfiability of Equation (11) unless $\mathcal{P}$ can generate non-trivial integers $\left\{a_{i}\right\}$ and $a$ satisfying $\tilde{g}^{a} \prod_{i=1}^{n} \tilde{g}_{i}{ }^{a_{i}}=1$.

If Equation (12) holds, then

$$
1=\frac{g^{\alpha} \prod_{i=1}^{n} g_{i}^{\alpha_{i}}}{g^{\prime}} \prod_{j=1}^{n}\left(\frac{g^{r_{j}} \prod_{i=1}^{n} g_{i}{ }^{A_{i j}}}{g_{j}^{\prime}}\right)^{c_{j}} \bmod p .
$$

If first two equations on Equations (17) does not hold, then the probability that Equation (12) hold is negligible. The same thing can be said for $m^{\prime},\left\{m_{i}^{\prime}\right\}_{(i=1, \ldots, n)}$ from the satisfiability of (13).

\section{C.2 Zero-Knowledge}

Theorem 10. We can construct a simulator of Main Protocol such that if there is a distinguisher who can distinguish between a real transcript from the protocol and an output from the simulator, then we can solve the decisional DiffieHellman problem.

A sketch of Proof: We first give a construction of the simulator. We then prove that if there exists such a distinguisher then we can solve $D D H_{n+1}^{5}$. From Lemma 1 and2, it means it is equivalent to solving the decisional Diffie-Hellman problem.

\section{The Construction of the Simulator}

We will construct the simulator $\mathcal{S}$ of the main protocol with the input $p, q, g, y, \tilde{g},\left\{\tilde{g}_{i}\right\},\left\{\left(g_{i}, m_{i}\right)\right\},\left\{\left(g_{i}^{\prime}, m_{i}^{\prime}\right)\right\}$ as follows. 
The simulator $\mathcal{S}$ first generates $s,\left\{s_{i}\right\},\left\{c_{i}\right\}, \lambda^{\prime} \in_{R} \mathbf{Z}_{\mathbf{q}}, t, v, w,\left\{u_{i}\right\},\left\{\dot{t}_{i}\right\}$, $\left\{\dot{v}_{i}\right\},\left\{\dot{w}_{i}\right\},\left\{\tilde{g}_{i}^{\prime}\right\} \in_{R} \mathbf{G}_{\mathbf{q}}$ randomly. Then it computes $\tilde{g}^{\prime}, g^{\prime}, m^{\prime}, u, \dot{v}, \dot{w}$ as the following.

$$
\begin{aligned}
u & =g^{\lambda^{\prime}} \prod_{j=1}^{n} u_{j}{ }^{-c_{j}^{2}} \bmod p \\
\tilde{g}^{\prime} & =\tilde{g}^{s} \prod_{j=1}^{n} \tilde{g}_{j}^{s_{j}} \tilde{g}_{j}^{\prime-c_{j}} \bmod p \\
g^{\prime} & =g^{s} \prod_{j=1}^{n}{g_{j}}^{s_{j}} g_{j}^{\prime-c_{j}} \bmod p \\
m^{\prime} & =y^{s} \prod_{j=1}^{n} m_{j}^{s_{j}} m_{j}^{\prime-c_{j}} \bmod p \\
\dot{v} & =t^{\lambda^{\prime}} v^{s} g^{\sum_{j=1}^{n}\left(s_{j}^{3}-c_{j}^{3}\right)} \prod_{j=1}^{n} \dot{t}_{j}^{-c_{j}^{2}} \dot{v}_{j}^{-c_{j}} \bmod p \\
\dot{w} & =w^{s} g^{\sum_{j=1}^{n}\left(s_{j}^{2}-c_{j}^{2}\right)} \prod_{j=1}^{n} \dot{w}_{j}^{-c_{j}} \bmod p .
\end{aligned}
$$

The output of $\mathcal{S}$ is

$\left(t, v, w, u,\left\{u_{i}\right\},\left\{\tilde{g}_{i}^{\prime}\right\}, \tilde{g}^{\prime}, g^{\prime}, m^{\prime},\left\{\dot{t}_{i}\right\},\left\{\dot{v}_{i}\right\}, \dot{v},\left\{\dot{w}_{i}\right\}, \dot{w},\left\{c_{i}\right\}, s,\left\{s_{i}\right\}, \lambda^{\prime}\right)$.

\section{A Distinguisher of $D_{n+1}^{5}$ and $R_{n+1}^{5}$}

We will then construct a distinguisher $\mathcal{D}^{\prime}$ who can distinguish between the uniform instances of $D_{n+1}^{5}$ and $R_{n+1}^{5}$ if $\mathcal{S}$ can not simulate the main protocol.

Let's say the instance $I$

$$
I=\left(x_{1}^{(1)}, x_{1}^{(2)}, \ldots, x_{1}^{(5)}, \ldots, x_{n+1}^{(1)}, x_{n+1}^{(2)}, \ldots, x_{n+1}^{(5)}\right)
$$

was chosen uniformly from either $D_{n+1}^{5}$ or $R_{n+1}^{5}$. Then this distinguisher will first generate $g_{1}, m_{1}, g_{2}, m_{2}, \ldots, g_{n}, m_{n}, \tilde{g}_{1}, \tilde{g}_{2}, \ldots, \tilde{g}_{n}$ as the constants used in Main Protocol and let $X \in_{R} \mathbf{Z}_{\mathbf{q}}, g=x_{1}^{(1)}, \tilde{g}=x_{1}^{(2)}, y=g^{X} \bmod p$. It will then generate a random permutation $A_{j i}$ and a secret key $X \in_{R} \mathbf{Z}_{\mathbf{q}}$ and compute

$$
\left(g_{i}^{\prime}, m_{i}^{\prime}\right)=\left(x_{i+1}^{(1)} \prod_{j=1}^{n} g_{j}^{A_{j i}},\left(x_{i+1}^{(1)}\right)^{X} \prod_{j=1}^{n} m_{j}^{A_{j i}}\right) \bmod p . \quad(i=1, \ldots, n)
$$

We note that $\left\{\left(g_{i}^{\prime}, m_{i}^{\prime}\right)\right\}$ gives a random shuffle of $\left\{\left(g_{i}, m_{i}\right)\right\}$.

Based on $g, y, \tilde{g},\left\{\tilde{g}_{i}\right\},\left\{\left(g_{i}, m_{i}\right)\right\}$ and $\left\{\left(g_{i}^{\prime}, m_{i}^{\prime}\right)\right\}$ the distinguisher $\mathcal{D}^{\prime}$ is going to act as a simulator $\mathcal{S}^{\prime}$ which simulates the simulator $\mathcal{S}$. More specifically, the simulator $\mathcal{S}^{\prime}$ randomly generates

$$
s,\left\{s_{i}\right\},\left\{c_{i}\right\}, \lambda^{\prime},\left\{\beta_{i}\right\} \in_{R} \mathbf{Z}_{\mathbf{q}} \quad i=1, \ldots, n
$$

and computes 


$$
\begin{aligned}
& t=x_{1}^{(3)}, v=x_{1}^{(4)}, w=x_{1}^{(5)} \\
& u_{i}=\left(x_{i+1}^{(1)}\right)^{\beta_{i}} \bmod p \quad i=1, \ldots, n \\
& u=g^{\lambda^{\prime}} \prod_{j=1}^{n} u_{j}^{-c_{j}^{2}} \bmod p \\
& \tilde{g}_{i}^{\prime}=x_{i+1}^{(2)} \prod_{j=1}^{n} \tilde{g}_{j}^{A_{j i}} \bmod p \quad i=1, \ldots, n \\
& \tilde{g}^{\prime}=\tilde{g}^{s} \prod_{j=1}^{n} \tilde{g}_{j}{ }^{s_{j}} \tilde{g}_{j}^{\prime-c_{j}} \bmod p \\
& g^{\prime}=g^{s} \prod_{j=1}^{n} g_{j}^{s_{j}} g_{j}^{\prime-c_{j}} \bmod p \\
& m^{\prime}=y^{s} \prod_{j=1}^{n} m_{j}^{s_{j}} m_{j}^{\prime}{ }^{-c_{j}} \bmod p \\
& \alpha_{j}=s_{j}-\sum_{k=1}^{n} A_{j k} c_{k} \bmod q \quad j=1, \ldots, n \\
& \dot{t}_{i}=\left(x_{i+1}^{(3)}\right)^{\beta_{i}} \prod_{j=1}^{n} g^{3 \alpha_{j} A_{j i}} \bmod p \quad i=1, \ldots, n \\
& \dot{v}_{i}=x_{i+1}^{(4)} \prod_{j=1}^{n} g^{3 \alpha_{j}^{2} A_{j i}} \bmod p \quad i=1, \ldots, n \\
& \dot{w}_{i}=x_{i+1}^{(5)} \prod_{j=1}^{n} g^{2 \alpha_{j} A_{j i}} \bmod p \quad i=1, \ldots, n \\
& \dot{v}=t^{\lambda^{\prime}} v^{s} g^{\sum_{j=1}^{n}\left(r_{j}^{3}-c_{j}^{3}\right)} \prod_{j=1}^{n}\left(\dot{t}_{j}^{-c_{j}^{2}} \dot{v}_{j}^{-c_{j}}\right) \bmod p \\
& \dot{w}=w^{s} g^{\sum_{j=1}^{n}\left(s_{j}^{2}-c_{j}^{2}\right)} \prod_{j=1}^{n} \dot{w}_{j}^{-c_{j}} \bmod p .
\end{aligned}
$$

The simulator $\mathcal{S}^{\prime}$ outputs

$\left(t, v, w, u,\left\{u_{i}\right\},\left\{\tilde{g}_{i}^{\prime}\right\}, \tilde{g}^{\prime}, g^{\prime}, m^{\prime},\left\{\dot{t}_{i}\right\},\left\{\dot{v}_{i}\right\}, \dot{v},\left\{\dot{w}_{i}\right\}, \dot{w},\left\{c_{i}\right\}, s,\left\{s_{i}\right\}, \lambda^{\prime}\right)$.

Lemma 9. Simulator $\mathcal{S}^{\prime}$ perfectly simulates Main Protocol when $I \in_{R} D_{n+1}^{5}$.

Sketch: We let

$$
\begin{aligned}
& \log _{x_{1}^{(1)}} x_{i+1}^{(1)}=r_{i}, \quad \log _{x_{1}^{(1)}}\left(x_{i+1}^{(1)}\right)^{\beta_{i}}=\lambda_{i} \\
& \log _{x_{1}^{(1)}} x_{1}^{(3)}=\tau, \quad \log _{x_{1}^{(1)}} x_{1}^{(4)}=\rho, \log _{x_{1}^{(1)}} x_{1}^{(5)}=\sigma .
\end{aligned}
$$

This gives for $i=1, . ., n$, 


$$
\begin{array}{r}
x_{i+1}^{(1)}=g^{r_{i}},\left(x_{i+1}^{(1)}\right)^{\beta_{i}}=g^{\lambda_{i}}, x_{i+1}^{(2)}=\tilde{g}^{r_{i}}, \\
\left(x_{i+1}^{(3)}\right)^{\beta_{i}}=g^{\tau \lambda_{i}}, x_{i+1}^{(4)}=g^{\rho r_{i}}, x_{i+1}^{(5)}=g^{\sigma r_{i}} .
\end{array}
$$

Therefore, it is clear that by randomly choosing $s,\left\{s_{i}\right\}, \lambda^{\prime}$ and $\left\{\beta_{i}\right\}$, it gives the same distribution of the output as when $\alpha,\left\{\alpha_{i}\right\},\left\{\lambda_{i}\right\}$ and $\lambda$ were first chosen randomly, and verifier honestly chooses random challenge $\left\{c_{i}\right\}$.

Lemma 10. Simulator $\mathcal{S}^{\prime}$ perfectly simulates $\mathcal{S}$ when $I \in_{R} R_{n+1}^{5}$

Sketch: Since $x_{i}^{(2)}, x_{i}^{(3)}, x_{i}^{(4)}, x_{i}^{(5)}(i=1,2, \ldots, n+1)$ and $\beta_{i}(i=1,2, \ldots, n)$ are randomly chosen, it gives the same distribution when $\tilde{g}, t, v, w,\left\{\tilde{g}_{i}^{\prime}\right\},\left\{\dot{t}_{i}\right\},\left\{\dot{v}_{i}\right\},\left\{\dot{w}_{i}\right\}$ and $\left\{u_{i}\right\}$ are randomly chosen for $i=1,2, \ldots, n$.

Therefore, if there exists a distinguisher $\mathcal{D}$ that distinguishes the output of the simulator $\mathcal{S}$ and a real transcript of Main Protocol, then this distinguisher can be used to solve $D D H_{n+1}^{5}$.

\section{Alternative Notation}

We present here an alternative notation of the variables. Since we have discussed the basis $\left\{g, g_{1}, \ldots, g_{n}\right\}$ throughout the paper, we can think of representing $g$ by $g_{0}$. Similarly $y$ by $m_{0}$ and $\tilde{g}$ by $\tilde{g}_{0}$. We can include the value of randomizers $\left\{r_{i}\right\}, \alpha_{i}$ and $\alpha$, in the matrix by defining $A_{0 i}=r_{i}, A_{i 0}=\alpha_{i}$, and $A_{00}=\alpha$.

Treating a public key in a similar manner with input variables may be awkward, but it gives a compact representation to some of the variables, e.g,

$$
g_{\mu}^{\prime}=\prod_{\nu=0}^{n} g_{\nu}^{A_{\nu \mu}}, m_{\mu}^{\prime}=\prod_{\nu=0}^{n} m_{\nu}^{A_{\nu \mu}}, \tilde{g}_{\mu}^{\prime}=\prod_{\nu=0}^{n} \tilde{g}_{\nu}^{A_{\nu \mu}} \quad \mu=0, \ldots, n .
$$

Further suggestions for the alternative notation follows:

$$
\begin{aligned}
& g_{0}^{\prime}=g^{\prime}, m_{0}^{\prime}=m^{\prime}, \tilde{g}_{0}^{\prime}=\tilde{g}^{\prime}, s_{0}=s, c_{0}=1, \lambda_{0}=\lambda, u_{0}=u \\
& s_{\mu}=\sum_{\nu=0}^{n} A_{\mu \nu} c_{\nu}, \lambda^{\prime}=\sum_{\nu=0}^{n} \lambda_{\nu} c_{\nu}^{2}, \prod_{\nu=0}^{n} g_{\nu}^{s_{\nu}}=\prod_{\nu=0}^{n} g_{\nu}^{\prime c_{\nu}}, g^{\lambda^{\prime}}=\prod_{\nu=0}^{n} u_{\nu}^{c_{\nu}^{2}} \quad \mu=0, \ldots, n .
\end{aligned}
$$

